\title{
Application of Viscoelastic testing to the field of Otolaryngology Head and Neck Surgery: A Scoping Review
}

Faisal Shariff', Mathew Marsee ${ }^{1}$, Mark Walsh $^{1,2}$

${ }^{1}$ Indiana University School of Medicine South Bend Campus, South Bend, Indiana, USA;

${ }^{2}$ Departments of Emergency and Internal Medicine, Saint Joseph Regional Medical Center, Mishawaka, Indiana, USA

\section{Background:}

In Otolaryngology Head and Neck Surgery (OHNS), hematologic management requires attention to the intricate nature of hemostatic competence. Elderly patients can experience drug induced coagulopathies. Strategies are needed in order to minimize hemorrhagic and thromboembolic risk and maximize positive outcomes. Viscoelastic tests (VETs) such as Thromboelastography (TEG) and Rotational Thromboelastometry (ROTEM) have been used to guide blood component therapy. The purpose of this scoping review is to understand the utilization of VETs in OHNS.

\section{Methods:}

This scoping review included studies that discussed the utilization of VETs in the field of OHNS. The inclusion criteria was studies that discussed the application of VETs in Flap/Plastic Reconstruction, Facial Trauma/Resuscitation, Tonsillectomy, Obstructive Sleep Apnea (OSA), Epistaxis, Sinus Surgery, and HNC Resection. Studies that focused on general medical VET use were not included.

\section{Results:}

Fourteen articles met the study inclusion criteria from an initial pool of 45 articles. Seven articles discussed the use of VETs in free flap/reconstructive surgery, five articles explored use in HNC, two articles discussed use in facial trauma and resuscitation, one article mentioned use in OSA, and one article examined use in epistaxis.

\section{Conclusion and potential impact:}

Of the fourteen articles included, two articles found no clinically significant association between TEG parameters and flap loss or complications. Five studies found that TEG or ROTEM helpful in predicting coagulation clotting, a prothrombotic state, or a hyperfibrinolytic state in free flap and HNC patients. For facial trauma and HNC cancer patients two different studies found ROTEM helpful in guiding blood component resuscitation. Using the TEG, one study demonstrated a hypercoagulable state in OSA patients. This review encourages wider use of VETs in OHNS in order to monitor hemostasis and improve surgical outcomes. Further research is necessary to better understand how VETs can tailor hematologic management of OHNS patients. 